\title{
A Particular Infantile Scimitar Syndrome Variant with Anomalous Systemic Arterial Supply-Inferior Vena Cava Fistula
}

\author{
Carmen Corina Șuteu ${ }^{1 *}$, Cristina Blesneac ${ }^{1}$, Marian Pop $^{2^{*}}$, Amalia Făgărășan², Liliana Gozar ${ }^{2}$, \\ Rodica Togănel ${ }^{2}$ \\ ${ }^{1}$ Department of Pediatric Cardiology, Emergency Institute for Cardiovascular Diseases and Transplantation, Târgu Mureș, Romania \\ 2 "George Emil Palade" University of Medicine, Pharmacy, Science and Technology, Târgu Mureș, Romania \\ ${ }^{*}$ Equal contribution
}

\section{ABSTRACT}

Scimitar syndrome is a rare congenital cardiovascular malformation that includes a partially anomalous drainage of the pulmonary veins in the inferior vena cava, right pulmonary hypoplasia, and systemic-pulmonary collaterals originating from various segments of the aorta. We present a case of Scimitar syndrome with associated intracardiac lesions and a large arterial conduct supplying the right lung, originating from the abdominal aorta and draining in the inferior vena cava.

Keywords: Scimitar syndrome, pediatric, aorta-inferior vena cava fistula

\section{ARTICLE HISTORY}

Received: February 10, 2021

Accepted: March 1, 2021

\section{CORRESPONDENCE}

\section{Cristina Blesneac}

Str. Gheorghe Marinescu nr. 50

540136 Târgu Mureș, Romania

Tel: +40 744794663

E-mail: cristinablesneac@gmail.com

\section{INTRODUCTION}

Scimitar syndrome is a rare cardiovascular and bronchopulmonary congenital abnormality, with an incidence of 1-5/100,000 live births. ${ }^{1,2}$ Scimitar syndrome consists of partial pulmonary venous drainage into the inferior vena cava (IVC), variable degrees of hypoplasia of the right lung with rotation of the heart into the right hemithorax, hypoplasia of the right pulmonary artery (PA), and an aberrant systemic arterial supply from the aorta to the right lung. ${ }^{3}$ The syndrome is variable in its expression, and several variants were described in the literature. ${ }^{4-6}$ Addi- tional intracardiac lesions have also been recognized, with a prevalence of $40 \%{ }^{2}$

\section{CASE REPORT}

We report the original case of a 6-weeks-old female infant who was transferred to our tertiary center, presenting with acute dyspnea and requiring admission in the pediatric intensive care unit and intubation. She was born at 36 weeks of gestation with a birth weight of 3,000 g. The physical examination showed respiratory distress, signs of congestive heart failure, and the lower limbs 


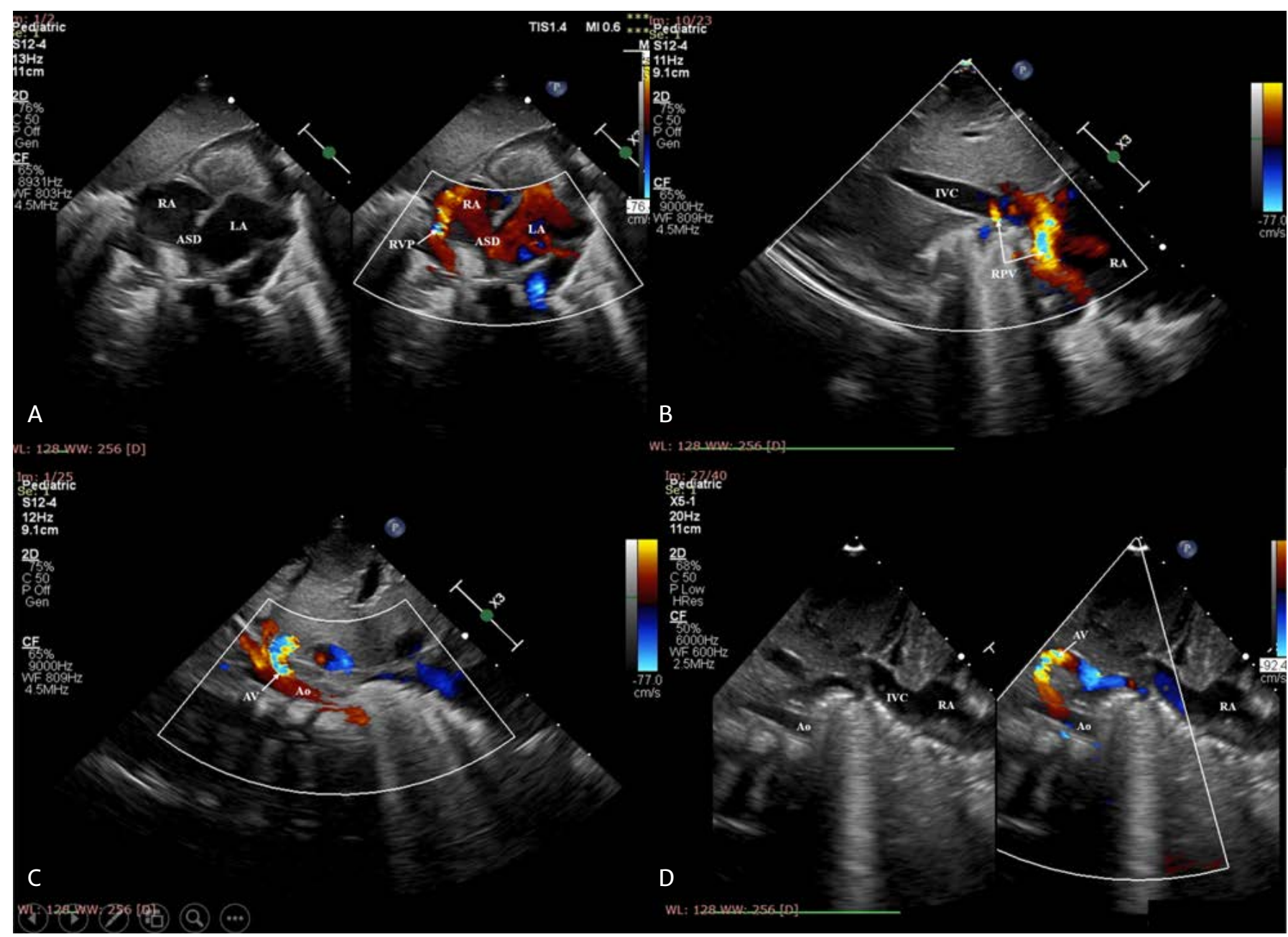

FIGURE 1. Color Doppler echocardiographic views demonstrating right pulmonary veins draining into the RA and IVC (A, B) and a large artery vessel arising from the abdominal aorta with ascending and tortuos course (C; D). Ao - abdominal aorta; ASD - atrial septal defect; AV - aberrant vessel; IVC - inferior vena cava; LA - left atrium; RA - right atrium

presented intermittent coldness. Cardiac auscultation revealed tachycardia with systolic murmur. Chest radiography revealed opacification of the right hemithorax, cardiac enlargement with moderate displacement of the cardiac structures from left to right, and pulmonary plethora; a scimitar vein was not observed. Echocardiography showed mesocardia, dilatation of the IVC, dilatation of both ventricles with normal function, ostium secundum interatrial communication (of $5 \mathrm{~mm}$ diameter) with left-to-right shunt, a muscular-type ventricular septum defect (of $4 \mathrm{~mm}$ diameter), hypoplasia of the right PA ( $z$ score: -2.4 ). Only the left pulmonary veins were recognized by echocardiography on the left side, a right pulmonary vein drained into the right atrium (RA), and a small pulmonary vein drained into the IVC (Figure 1, panels A and B). The subcostal view revealed a large arterial vessel arising from the abdominal aorta near the celiac axis, with an ascending and tortuous course (Figure 1 , panels $C$ and $D$ ).

The computed tomography scan revealed hypoplasia of the right lung, with right PA hypoplasia; no parenchyma appeared to be excluded from the normal bronchial tree (Figure 2, panel A). An anomalous venous return was observed, with a dilated right pulmonary vein opening into the RA, which drained the pulmonary venous circulation corresponding to the right upper and middle lobe, and the upper and anterobasal segments of the right lower lobe (Figure 2, panel B). Another pulmonary vein collecting the laterobasal and posterobasal segments of the right lower lobe was observed crossing the diaphragm and joining with another pulmonary vein that drained the mediobasal segment of the right lower lobe, with a common draining site into the IVC. There was a large aberrant artery arising from the abdominal aorta above the celiac trunk with as- 


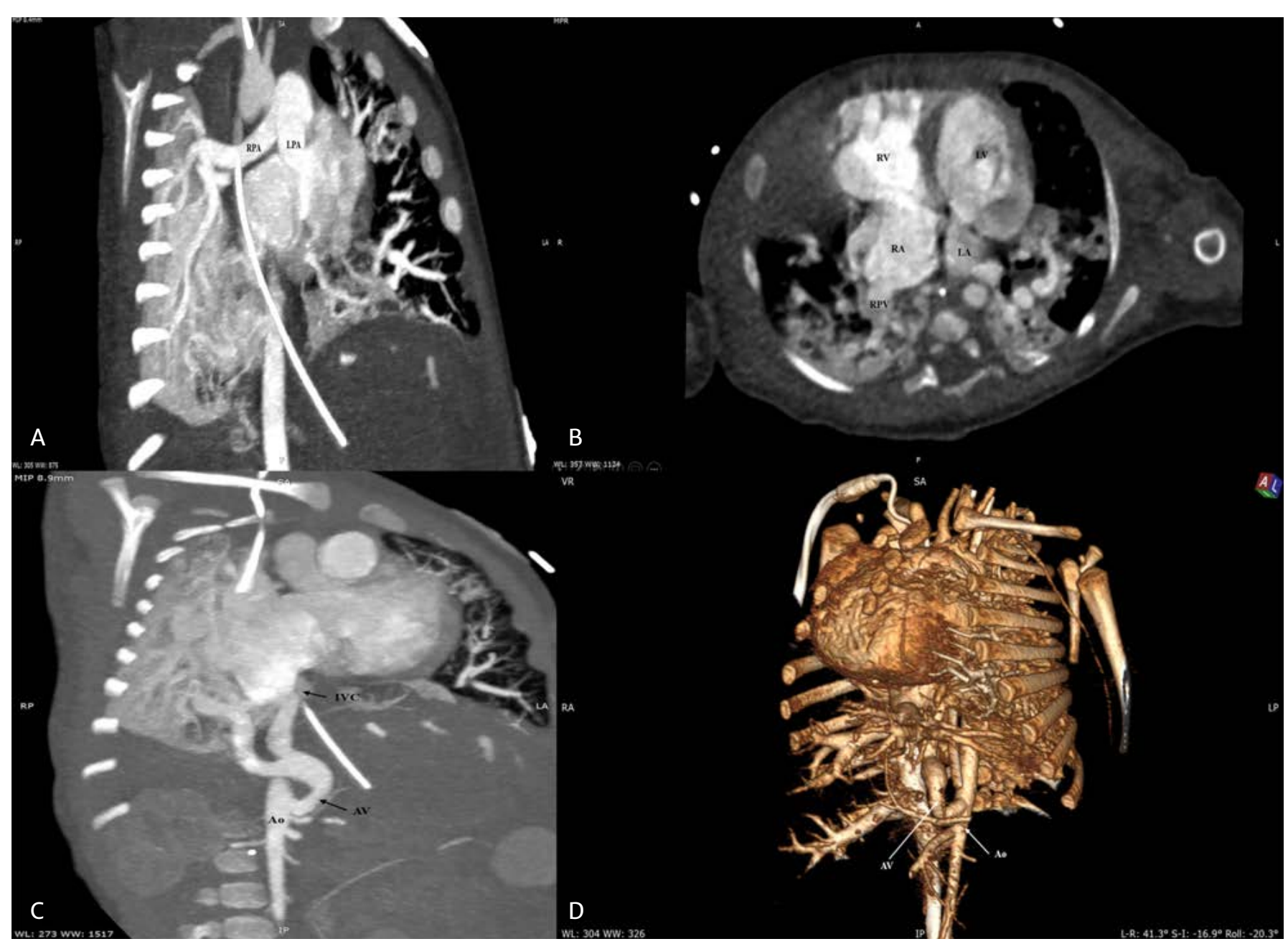

FIGURE 2. Computed tomography angiography scan showing a small right pulmonary artery (A), a right dilated pulmonary vein draining into the right atrium (B), and an aberrant vessel arrising from abdominal aorta supplying the right pulmonary circulation and fistulising in the inferior vena cava (C, D). Ao - abdominal aorta; AV - aberrant vessel; IVC - inferior vena cava; LA - left atrium; LPA - left pulmonary artery; LV - left ventricle; RA - right atrium; RPA - right pulmonary artery; RPV - right pulmonary vein; RV - right ventricle

cending, transdiaphragmatic course, which separated into two branches, one supplying the lower part of the right pulmonary circulation, and the other draining into the cranial portion of the IVC, which was actually an aortocaval fistula (Figure 2, panels C and D). Below the origin of this artery, the aorta was narrowed.

Cardiac catheterization confirmed the diagnosis of Scimitar variant, with the presence of a systemic-to-pulmonary collateral vessel arising from abdominal aorta below the diaphragm, which supplied an important area of the right lung and from which an aorto-venous fistula had developed, draining into the IVC (Figure 3). The hemodynamics revealed a mean pulmonary arterial pressure of $32 \mathrm{mmHg}$, a systemic-pulmonary output ratio (Qp:Qs) of 7.82, a pulmonary/systemic vascular resistance ratio (Rp:Rs) of 0.2 , an oxygen saturation in the IVC of $55.8 \%$ at the level of the lower margin of the liver and $94.4 \%$ at the level of RA.
In spite of vigorous treatment for congestive heart failure, the condition of the infant deteriorated. The patient underwent surgery on the 12th day after admission to suppress flow through the aberrant vessel. After the patient had been put on cardiopulmonary bypass, the anomalous vessel was identified and ligated immediately after its emergence. Following the procedure, there was an improvement in the infant's condition. Postoperative echocardiography estimated a normal pressure in pulmonary circulation and demonstrated left-to-right shunt at three levels: at atrial and ventricular level, and at the partial anomalous pulmonary venous return, with a Qp:Qs of 1.8:1. The anomalous venous return will be surgically treated later in evolution.

The publication of this case was approved by the institutional review board of the Emergency Clinical County Hospital of Târgu Mureș, in the presence of a signed informed consent obtained from the mother. 


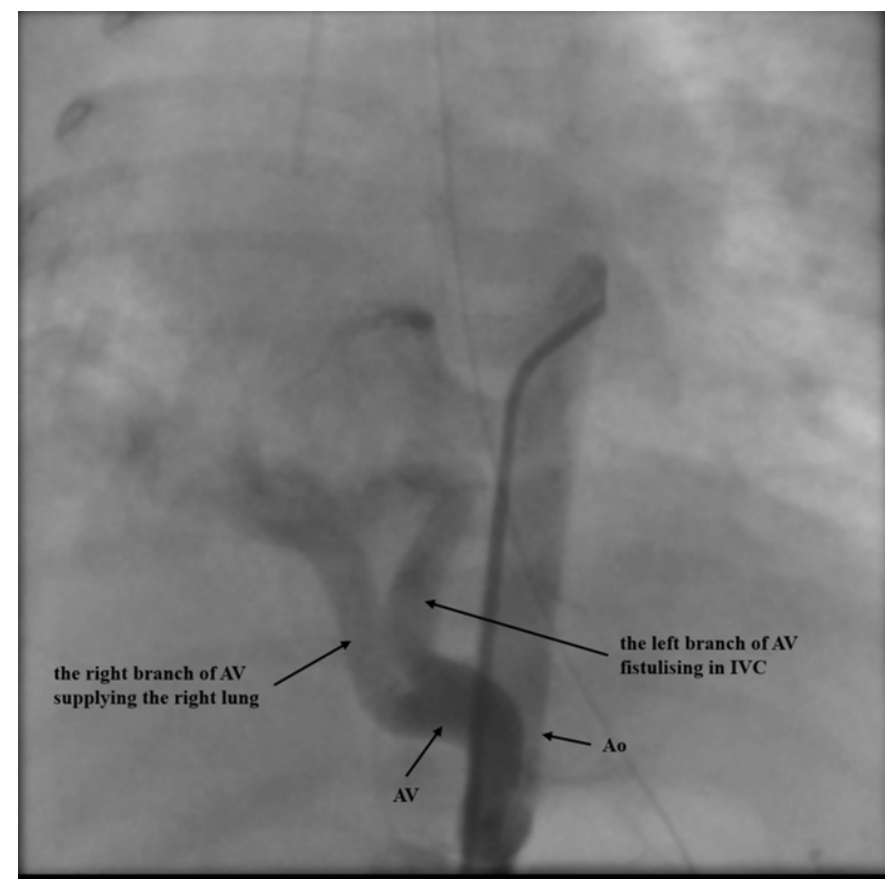

FIGURE 3. Selective arteriogram illustrating the aberrant large artery arising from the aorta supplying the right lung and fistulising in the inferior vena cava. Ao - abdominal aorta; AV - aberrant vessel; IVC - inferior vena cava

\section{DISCUSSIONS}

Scimitar syndrome was classified by Depuis et al. into two distinct forms: "infantile" and "adult". 3 The "infantile" form affects children younger than one year of age, and it typically presents the clinical features of pulmonary arterial hypertension and congestive heart failure secondary to the (1) significant left-to-right shunt from the anomalous pulmonary venous drainage, from an additional cardiac defect, and from the systemic arterial supply to the right lung; and (2) reduction of the pulmonary vascular bed on the right side., The "adult" form, presenting without pulmonary hypertension, affects both children and adults, is well tolerated, and has a better prognosis. $^{3}$

The management of Scimitar syndrome is diverse due to the variability of its clinical and hemodynamic characteristics. The operative approach should be individualized, and a two-stage approach has been recommended. ${ }^{3}$ Embolization through a catheter or surgical ligation of the aberrant arterial supply as the first operative phase has been shown to improve the clinical features of congestive heart failure by reducing the left-to-right shunt., ${ }^{1,7,8}$

In our case, ligation of the anomalous systemic arterial collateral immediately after its origin reduced the left-toright shunt by eliminating the right pulmonary circulation supply and removing the aorta-IVC fistula.
The unique pattern of the presented case can be explained in the light of proper knowledge of the embryological development of the cardiovascular system. In early fetal life, there is an intercommunication between developing cardiac chambers and systemic, pulmonary, and venous systems. The persistent communication of the splanchnic plexus with a cardinal or umbilic-vitelline vein may lead to the development of some type of anomalous venous connection. ${ }^{9}$ If early connections of both arteries and veins persist, a congenital arteriovenous fistula may result. Le Rochais et al. reported a Scimitar syndrome associated with pulmonary arteriovenous fistulas. ${ }^{10}$ According to the authors' knowledge, this is the first case published in the literature, which associates these particular anatomical features, consisting in the association of an aorta-IVC fistula in a patient with Scimitar syndrome without pulmonary sequestration.

\section{CONCLUSIONS}

Infantile Scimitar syndrome is a rare congenital anomaly that is difficult to diagnose and manage. Our case represents a unique variant of the Scimitar syndrome with a normal bronchial tree, associated intracardiac malformation, and an anomalous right pulmonary arterial supply originating from the abdominal aorta, with a fistula into the IVC.

\section{CONFLICT OF INTEREST}

Nothing to declare.

\section{REFERENCES}

1. Depuis C, Charaf LA, Breviere GM, Abou P. "Infantile" form of Scimitar syndrome with pulmonary hypertension. Am J Cardiol. 1993;71:1326-1330. doi: 10.1016/0002-9149(93)90549-r.

2. Rukban HAI, Ghaihab MAI, Tamini O, Al-Saleh S. Clinical spectrum of infantile scimitar syndrome: A tertiary center experience. Ann Pediatr Cardiol. 2014;7:29-33. doi: 10.4103/0974-2069.126547.

3. Dupuis C, Charaf LA, Breviere GM, Abou P, Remy-Jardin M, Helmius G. The "adult" form of the Scimitar syndrome. Am J Cardiol. 1992;70:502-507. doi: 10.1016/0002-9149(92)91198-d.

4. Argueta-Morales IR, Meador LC, Nykanen DG, DeCampli WM. Infantile form of scimitar syndrome with collateral pulmonary vein stenosis. Pediatr Cardiol. 2010;31:550-552. doi: 10.1007/ s00246-009-9630-z.

5. Babazadeh K, Zamani H, Ghaemi H, Omran FA. A case of variant scimitar syndrome. Caspian Journal of Pediatrics. 2017;17:241-244. doi: 10.22088/acadpub.BUMS.3.2.253.

6. Bo I, Daubeney PEF, Rigby ML. Uncommon variants of the Scimitar syndrome in two siblings. Annals of Pediatric Cardiology. 2015;8:56-58. doi: 10.4103/0974-2069.149523. 
7. Uthaman B, Abushaban L, Al-Qbandi M, Rathinasamy J. The impact of interruption of anomalous systemic arterial supply on scimitar syndrome presenting during infancy. Catheter Cardiovasc Interv. 2008;71:671-678. doi: 10.1002/ccd.21430.

8. Brown JW, Ruzmetov M, Minnich DJ, et al. Surgical management of Scimitar syndrome: An alternative approach. J Thorac Cardiovasc Surg. 2003;125:238-245. doi: 10.1067/ mtc.2003.113.
9. Ghandour A, Partovi S, Karappasamy K, Rajiah P. Congenital anomalies of the IVC-embriological perspectives and clinical relevance. Cardiovasc Diagn Ther. 2016;6:482-492. doi: 10.21037/cdt.2016.11.18.

10. Le Rochais JP, Icard P, Davani S, Abouz D, Evrard C. Scimitar Syndrome with pulmonary arteriovenous fistulas. Ann Thorac Surg. 1999;68:1416-1418. doi: 10.1016/s0003-4975(99)00731-6. 